\title{
Sequential therapy for Helicobacter pylori infection with and without probiotics
}

\begin{abstract}
Background: Helicobacter pylori (H. pylori) infection is considered to be among the most common infections all around the world. Previous studies have shown that H. pylori is gaining resistance to several antibiotic agents. This prompted the clinicians to prescribe various combined therapies, including, probiotics to overcome the drug resistance. The aim of this study was to determine whether probiotics can increase the eradication rate of $H$. pylori infection.
\end{abstract}

Materials and methods: This control trial was carried out on 106 H. pylori positive patients, assigned into two groups. Group A was treated with Amoxicillin, Pantoprazole, Tinidazol and Clarithromycin; group B was treated Familact capsules consisted of probiotics added to the same regimen administered to group A. At the end of treatment, urease breath test was done for the participants, and reported as positive or negative. T-test and Chi-square test were used for evaluation of the results, and P-value $<0.05$ was considered as significant.

Results: $H$. pylori eradication rate in group B (88.5\%) was significantly higher than group A $(63.3 \%)$. Besides, the participants who received probiotics seemed to show lower side effects, including, bad taste and epigastric pain.

Conclusion: The addition of probiotics to the sequential therapy could significantly increase $H$. pylori eradication rate.

\author{
Volume II Issue 6 - 2020
}

\begin{abstract}
Rahmatollah Rafiei, 'Alireza Ebrahimi, ${ }^{2}$ Mahmood Bahrami, ${ }^{3}$ Maryam Nazari,' Zahra Torabi, ${ }^{4}$ Armin Ebrahimi,' Fereshteh Rafiei,' Sahar Hosseini, ${ }^{2}$ Soheil Ashkani-Esfahani ${ }^{5}$ 'Department of Internal Medicine, Islamic Azad University, Iran ${ }^{2}$ Student Research Committee, Shiraz University of Medical sciences, Iran

${ }^{3}$ Medical School, Isfahan University of Medical Sciences, Iran ${ }^{4}$ School of Medicine, St. George University, England ${ }^{5}$ Department of Orthopaedic Surgery, Massachusetts General Hospital, USA
\end{abstract}

\section{Correspondence: Alireza Ebrahimi, Student Research Committee, Medical School, Shiraz University of Medical sciences, Karimkhan Zand St., Shiraz, Iran, Emailmd.alirezaebrahimi@gmail.com}

Received: November 03, 2020 | Published: December 16, 2020

Keywords: helicobacter pylori, sequential therapy, probiotics

\section{Introduction}

Helicobacter pylori (H. pylori) infection is considered to be the most frequent infection involving gastrointestinal (GI) system, worldwide. The prevalence of H. pylori infection is measured to be 70 $90 \%$ in the developing countries, and $25-50 \%$ in the developed areas. ${ }^{1}$ The disease can also lead to several upper GI diseases, including, chronic gastritis, and gastric malignancies. ${ }^{2}$ These facts, besides, the problem of raising antibiotic resistance had prompted the clinicians to vigorously try to find a more effective therapy for the infection. Sequential therapy is among the most common recommended treatment for $H$. pylori infection, and is consisted of a 10-days course of proton pump inhibitor (PPI), plus, 3 proper antibiotics, given in sequence. ${ }^{3}$ Previous studies showed that this regimen could lead to higher rates of $H$. pylori eradication in comparison with the conventional triple therapy. ${ }^{4-7}$ However, the antibiotic-associated GI side effects, including, nausea, diarrhea, bloating, vomiting, and abdominal pain, are considered among the major problems of the $H$. pylori therapies. ${ }^{8,9}$

Probiotics are microorganisms that decrease the harmful bacteria, and improve the intestinal microenvironment for digestion. These microorganisms could play a role in preventing and even eradicating H. pylori infection, according to the current evidence. ${ }^{10}$ As supplemental probiotics increased the eradication rates of $H$. pylori in previous investigations. ${ }^{11,12}$ While, there is still controversy in this regard, and some reports mentioned that the addition of probiotics does not have any significant impact on the eradication rates of $H$. pylori infection. ${ }^{13,14}$ Furthermore, the administration of probiotics was beneficial for decreasing the side effects of therapies, and increasing the treatment tolerance. ${ }^{12,15}$

Several studies have mentioned the positive outcomes of adding probiotics to standard triple therapy., ${ }^{8,12,15-23}$ While, the effect of adding probiotic to sequential therapy was not yet clearly investigated. In the present study, we aimed to evaluate the impacts of adding probiotics to the sequential therapy, and the side effects of this regimen.

\section{Material and methods}

This randomized-control trial was carried out on $106 \mathrm{H}$. pylori positive patients enrolled between June 2018 to June 2019 in Shariati hospital, Isfahan, Iran. The patients who underwent endoscopy because of different indications, and had positive H. pylori were considered eligible for the study. ${ }^{24}$ The inclusion criteria were as following: 1. Agreement on informed consent to participate in the study; 2 . Age between 18 to 90 years old; 3 . Not having used H. pylori eradication treatments prior to the investigation; and 4. Not having used the antibiotics that are prescribed in the investigation, over the last month.

The exclusion criteria were the patients' unwillingness to enroll in the study or continue the treatment, not having the medical records of the patients, or comorbidities that affect the treatment process. The patients were enrolled in the study using available sampling method, and were randomly assigned into two groups. The group A was treated with Amoxicillin 1000 mg (two 500mg Capsule, Daana Pharma ${ }^{\mathrm{TM}}$, 
Iran) and Pantoprazole 40mg (Capsule, Abidi Pharma ${ }^{\mathrm{TM}}$, Iran) twice a day for 5 days, then Pantoprazole $40 \mathrm{mg}$, Tinidazol $500 \mathrm{mg}$ (Tablet, Abidi Pharma ${ }^{\mathrm{TM}}$, Iran), and Clarithromycin 500mg (Tablet, Daana Pharma ${ }^{\mathrm{TM}}$, Iran) twice a day for 5 days aftermath. The same regimen was also used for the treatment of group $\mathrm{B}$, with the addition of supplemental probiotic capsules (500mg, Familact, Capsule, Zist TakhmirTM, Iran), consisting of Lactobacillus rhamnosus, Lactobacillus casei, Streptococcus thermophiles, Lactobacillus bulgaricus, Lactobacillus acidophilus, Bifidobacterium breve, and Bifidobacterium longum, twice a day for 10days. Urea breath test (UBT; Heliprobe ${ }^{\circledR}$ system, Sweden) was performed after 8 hours of fasting by a gastroenterologist for the participants, 1 week after 10days treatment period. The patients were visited during and after the treatment process, in order to evaluate any improvement of side effects of the regimen by a person who was blinded to the study.

The protocol of this investigation was approved by the medical ethics committee of the Medical School of Islamic Azad University of Najafabad, Iran (IRB number: Reg. No: ir.iau.najafabad.rec.1396.79). All results documented and analyzed by SPSS Software version 22.0. Data were depicted as means \pm SD and also frequency. Demographic data were analyzed by using chi-square and t test wherever appropriate. In order to compare data within and between the groups, student $t$ test and paired t test were used, respectively. P-values less than 0.05 were considered as significant. The analysis of covariance test was applied for eliminating the impact of confounding parameters, both in the beginning and during the investigation.

\section{Results}

52 out of 53 patients in group B (98.1\%), and the entire patients in group A (100\%) had completed the study. The exclusion of one participant from group B did not affect the study process, and the results of the tests. Demographic characteristics of the participants were summarized in Table 1. The group B was consisted of 25 (47.2\%) male, and 28 (52.8\%) female patients; and group A had 21 $(31.6 \%)$ male, and $32(60.4 \%)$ female patients. All participants were aged between 20 and 89 years old. There was no association between the eradication rate of $H$. pylori and the participants' gender ( $\mathrm{p}=$ $0.43)$ or age $(\mathrm{p}=0.14)$. H. pylori eradication rate in group $\mathrm{B}(88.5 \%$; $\mathrm{n}=46)$ was significantly higher than group A $(63.3 \% ; \mathrm{n}=34)$ in group A $(\mathrm{p}=0.003)$. Figure 1 compares the eradication rate of $H$. pylori between the two groups.

Table I Demographic data

\begin{tabular}{lllll}
\hline \multirow{2}{*}{ Variables } & \multicolumn{4}{l}{ Groups } \\
\cline { 3 - 5 } & A & B & $\mathbf{P}_{\text {value }}{ }^{*}$ \\
\hline \multirow{2}{*}{ Gender } & Male & $39.6 \%(21)$ & $47.2 \%(25)$ & \\
& Female & $60.4 \%(32)$ & $52.8 \%(28)$ & 0.43 \\
Age & & $42 \pm 12.7$ & $46 \pm 16$ & 0.14 \\
\hline
\end{tabular}

*PValue $<0.05$ was considered as statistically significant

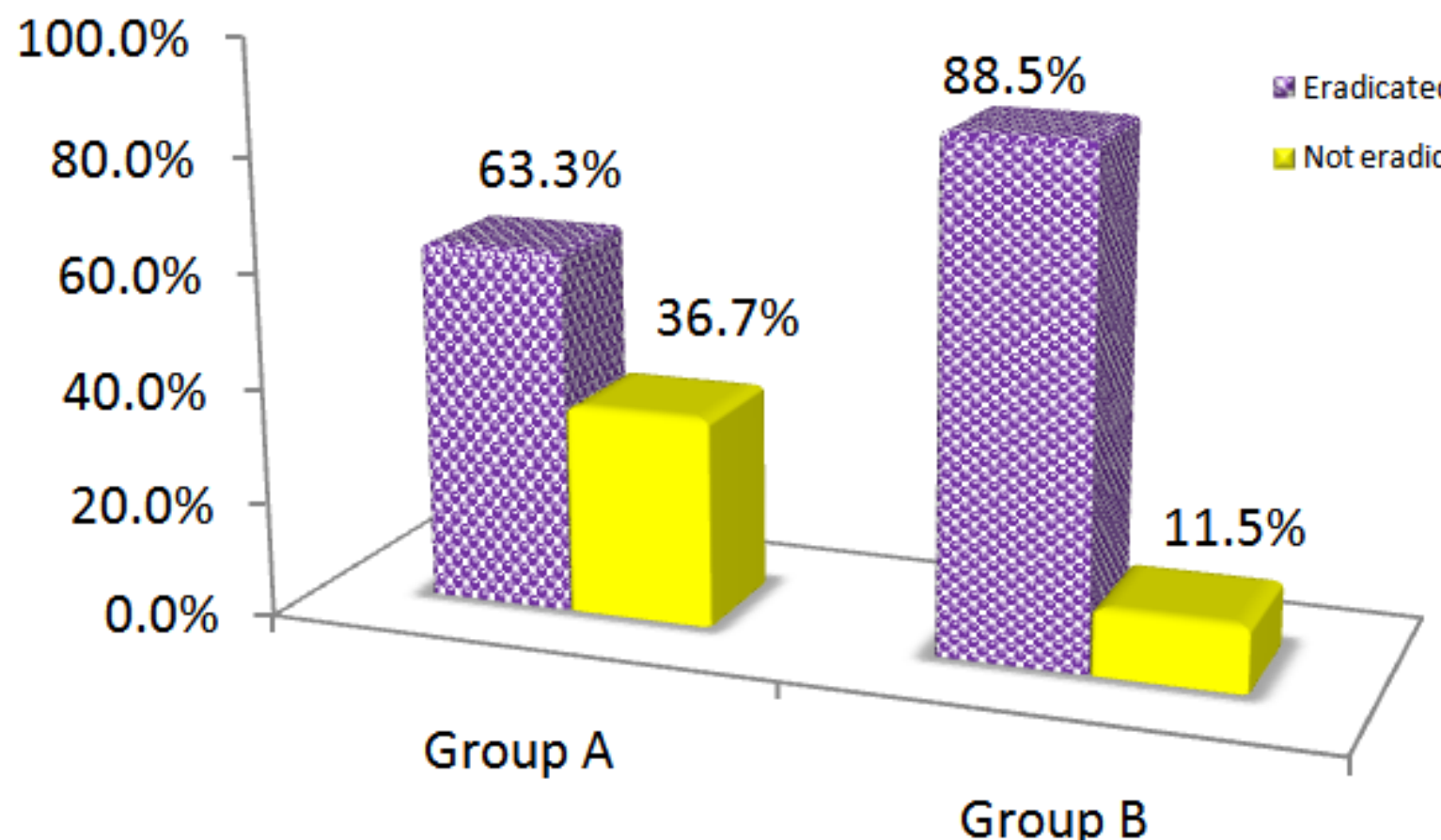

Figure I H. pylori eradication rate in Group A and Group B. Group B shows significantly higher H. pylori eradication rates, compared to group A ( $P<0.05)$.

Considering the side effects of sequential therapy, there were no significant differences in occurrence of headache, dizziness, itching, anorexia, bloating, fatigue, rash, nausea, vomiting, and constipation between the two groups $(\mathrm{P}=0.09)$. However, bad taste, and epigastric pain were considerably lower in group B compared to group A $(\mathrm{p}=0.005$, and $\mathrm{p}=0.007)$. All the above-mentioned side effects were found to be mild, and well tolerated (Table 2) 
Table 2 Side effects of sequential therapy in participants

\begin{tabular}{|c|c|c|c|c|c|c|c|c|c|c|c|c|}
\hline & $\begin{array}{l}\text { Side } \\
\text { effect }\end{array}$ & Headache & Dizziness & $\begin{array}{l}\text { Bad } \\
\text { taste }\end{array}$ & Itching & $\begin{array}{l}\text { Epigastric } \\
\text { pain }\end{array}$ & Anorexia & Bloating & Fatigue & Rash & $\begin{array}{l}\text { Nausea \& } \\
\text { vomiting }\end{array}$ & Constipation \\
\hline Group A & 31 & 2 & 1 & 17 & 1 & 0 & 1 & 2 & 2 & 1 & 4 & 0 \\
\hline Group B & 12 & 0 & 0 & 5 & 0 & 3 & 0 & 0 & 0 & 0 & 2 & 2 \\
\hline $\mathrm{P}_{\text {value }}{ }^{*}$ & 0.00 & 0.15 & 0.32 & 0.005 & 0.32 & 0.007 & 0.32 & 0.15 & 0.15 & 0.32 & 0.41 & 0.14 \\
\hline
\end{tabular}

\section{Discussion}

In our study, adding probiotics to the sequential therapy significantly enhanced the eradication rates of $H$. pylori. The result of present study is consistent with prior findings in this regard, as the 8-weeks administration of Lactobacillus gasseri was found to reduce $H$. pylori infection, and inflammation of gastric mucosa. ${ }^{25}$ Lactobacillus johnsonii was also had modulating impact on the $H$. pylori colonization in the pediatrics. ${ }^{26}$ Moreover, it has been mentioned that sixteen-weeks prescription of Lactobacillus johnsonii had decreased the H. pylori density, and the severity of antral gastritis. ${ }^{27}$ The addition of Bifidobacterium longum to the standard triple therapy was also resulted in higher eradication rates of $H$. pylori infection. ${ }^{28}$ Saccharomyces boulardii supplementation had also increased the H. pylori eradication rate. ${ }^{29}$ In this regard, a recent meta-analysis, consisted of 1469 subjects, reported that probiotic supplementation for patients who receive $H$. pylori therapies could significantly improve the eradication rate. ${ }^{30}$

The present study showed that adding probiotics to the sequential therapy could reduce the side effects of the treatment, as well. This also is consistent with prior evidence, since the addition of Lactobacillus rhamnosus $G G$ to the standard triple therapy had reduced the occurrence of GI side effects. ${ }^{15}$ Besides, Bifidobacterium longum supplementation had reduced the frequency of diarrhea in the patients. ${ }^{28}$ Previous comprehensive investigations also mentioned that probiotic supplementation could decrease the occurrence of side effects, and increase the treatment tolerance by the patients. ${ }^{12,29-31}$

In the present investigation we prescribed a supplemental capsule of probiotics, consisting of Lactobacillus rhamnosus, Lactobacillus casei, Streptococcus thermophiles, Lactobacillus bulgaricus, Lactobacillus acidophilus, Bifidobacterium breve, and Bifidobacterium longum for the patients, which showed promising results. It has been mentioned that probiotic supplementation might reinforce gut innate defenses; as in vitro studies showed that Lactobacillus acidophilus could decrease the adherence of $H$. pylori to the enterocytes. ${ }^{32}$ Considering the different mechanisms of various strains of probiotics, researchers suggested that combination of distinctive strains could be useful for increasing the eradication rate of infection. ${ }^{33,34}$ However, more comprehensive studies mentioned that although multi-strain adjuvant probiotics can be beneficial as an additional therapy, combined probiotics could not significantly increase the efficacy. ${ }^{29,31}$

Not measuring the recurrence rate of infection in the participants to evaluate the long-term effects of the treatments is one of the limitations of this study. Moreover, not classifying the patients to age groups, and relatively low number of participants are the other limitations that could be mentioned. Another limitation is that the results may not apply to other countries and populations and further investigations on the effectiveness of these regimens should be performed on similar populations in different regions.

\section{Conclusion}

This study showed that the addition of combined probiotics to the sequential therapy could significantly increase the eradication rate of H. pylori, and reduce the side effects of treatment, particularly, bad taste and epigastric pain. However, further clinical investigations are still necessary to find the definite efficacy of the approach.

\section{Declarations}

\section{Ethics approval and consent to participate}

Informed consent was obtained from all participants, their anonymity was guaranteed, and the study protocol was approved by the institutional research board (IRB) of Islamic Azad University of Najafabad, Isfahan, Iran (Reg. No: ir.iau.najafabad.rec.1396.79).

\section{Consent for publication}

All authors of this article have been involved with the investigation and have approved the paper and agree to its submission and publishing in this journal.

\section{Availability of data and material}

The datasets used during the current study are available from the corresponding author on reasonable request.

\section{Competing interest}

The authors have no competing interest to declare.

\section{Funding}

The authors did not receive any financial help for this investigation.

\section{Acknowledgments}

Authors want to thank Medipress Co. and SIMR Co. for helping us in preparing and editing the draft of the paper and helping in finalizing the article.

\section{References}

1. Go M. Natural history and epidemiology of Helicobacter pylori infection. Aliment Pharmacol Ther. 2002;16(Suppl 1):3-15.

2. Uemura N, Okamoto S, Yamamoto S, et al. Helicobacter pylori infection and the development of gastric cancer. N Engl J Med. 2001;345(11):784 789.

3. Chung JW, Jung YK, Kim YJ, et al. Ten-day sequential versus triple therapy for $\mathrm{H}$ elicobacter pylori eradication: A prospective, open-label, randomized trial. Gastroenterology. 2012;27(11):1675-1680.

4. Sardarian H, Fakheri H, Hosseini V, et al. Comparison of Hybrid and Sequential Therapies for $\mathrm{H}$ elicobacter pylori Eradication in I ran: A Prospective Randomized Trial. Helicobacter. 2013;18(2):129-134. 
5. Jafri NS, Hornung CA, Howden CW. Meta-analysis: sequential therapy appears superior to standard therapy for Helicobacter pylori infection in patients naive to treatment. Ann Intern Med. 2008;148(12):923-931.

6. Vaira D, Zullo A, Vakil N, et al. Sequential therapy versus standard tripledrug therapy for Helicobacter pylori eradication: a randomized trial. Ann Intern Med. 2007;146(8):556-563.

7. Gatta L, Vakil N, Leandro G, et al. Sequential Therapy or Triple Therapy for Helicobacter pylori Infection: Systematic Review and MetaAnalysis of Randomized Controlled Trials in Adults and Children. Am J Gastroenterol. 2009;104(12):3069-3079.

8. Bell G, Powell K, Burridge S, et al. Experience with 'triple'antiHelicobacter pylori eradication therapy: side effects and the importance of testing the pre-treatment bacterial isolate for metronidazole resistance. Antimicrob Agents Chemother. 1992;6(4):427-435.

9. Armuzzi A, Cremonini F, Ojetti V, et al. Effect of Lactobacillus GG Supplementation on Antibiotic-Associated Gastrointestinal Side Effects during Helicobacter pylori Eradication Therapy: A Pilot Study. Digestion. 2001;63(1):1-7.

10. Deguchi R, Nakaminami H, Rimbara E, et al. Effect of pretreatment with Lactobacillus gasseri OLL2716 on first-line Helicobacter pylori eradication therapy. J Gastroenterol Hepatol. 2012;27(5):888-892.

11. Hamilton-Miller J. The role of probiotics in the treatment and prevention of Helicobacter pylori infection. International Journal of Antimicrobial Agents. 2003;22(4):360-366.

12. Tong J, Ran Z, Shen J, et al. Meta-analysis: the effect of supplementation with probiotics on eradication rates and adverse events during Helicobacter pylori eradication therapy. Alimentary Pharmacology \& Therapeutics. 2007;25(2):155-168.

13. Szajewska H, Albrecht P, Topczewska-Cabanek A. Randomized, doubleblind, placebo-controlled trial: effect of lactobacillus GG supplementation on Helicobacter pylori eradication rates and side effects during treatment in children. Journal of pediatric gastroenterology and Nutrition. 2009;48(4):431-436.

14. Akcam M, Koca $\mathrm{T}$, Salman $\mathrm{H}$, et al. The effects of probiotics on treatment of Helicobacter pylori eradication in children. Saudi Med J. 2015;36(3):286-290.

15. Armuzzi A, Cremonini F, Ojetti V, et al. Effect of Lactobacillus GG supplementation on antibiotic-associated gastrointestinal side effects during Helicobacter pylori eradication therapy: a pilot study. Digestion. 2001;63(1):1-7.

16. Goldman CG, Barrado DA, Balcarce N, et al. Effect of a probiotic food as an adjuvant to triple therapy for eradication of Helicobacter pylori infection in children. Nutrition. 2006;22(10):984-988.

17. Nista EC, Candelli M, Cremonini F, et al. Bacillus clausii therapy to reduce side-effects of anti-Helicobacter pylori treatment: randomized, double-blind, placebo controlled trial. Aliment Pharmacol Ther. 2004;20(10):1181-1188.

18. Cremonini F, Di Caro S, Covino M, et al. Effect of different probiotic preparations on anti-Helicobacter pylori therapy-related side effects: a parallel group, triple blind, placebo-controlled study. Am J Gastroenterol. 2002;97(11):2744-2749.

19. Sheu BS, Cheng HC, Kao AW, et al. Pretreatment with Lactobacillus-and Bifidobacterium-containing yogurt can improve the efficacy of quadruple therapy in eradicating residual Helicobacter pylori infection after failed triple therapy. Am J Clin Nutr. 2006;83(4):864-869.
20. Tursi A, Brandimarte G, Giorgetti GM, et al. Effect of Lactobacillus casei supplementation on the effectiveness and tolerability of a new secondline 10-day quadruple therapy after failure of a first attempt to cure Helicobacter pylori infection. Med Sci Monit. 2004;10(12):CR662-CR6.

21. Duman DG, Bor S, Özütemiz Ö, et al. Efficacy and safety of Saccharomyces boulardii in prevention of antibiotic-associated diarrhoea due to Helicobacterpylori eradication. Eur J Gastroenterol Hepatol. 2005;17(12):1357-1361.

22. Canducci F, Armuzzi A, Cremonini F, et al. A lyophilized and inactivated culture of Lactobacillus acidophilus increases Helicobacter pylori eradication rates. Aliment Pharmacol Ther. 2000;14(12):1625-1629.

23. Myllyluoma E, Veijola L, Ahlroos T, et al. Probiotic supplementation improves tolerance to Helicobacter pylori eradication therapy-a placebo-controlled, double-blind randomized pilot study. Alimentary Pharmacology \& Therapeutics. 2005;21(10):1263-1272.

24. Bacon B. Harrison's Principles of Internal Medicine. 19e. New York, NY: McGraw-Hill Education; 2015.

25. Sakamoto I, Igarashi M, Kimura K, et al. Suppressive effect of Lactobacillus gasseri OLL 2716 (LG21) on Helicobacter pylori infection in humans. Journal of Antimicrobial Chemotherapy. 2001;47(5):709-710.

26. Cruchet S, Obregon MC, Salazar G, et al. Effect of the ingestion of a dietary product containing Lactobacillus johnsonii La1 on Helicobacter pylori colonization in children. Nutrition. 2003;19(9):716-721.

27. Pantoflickova D, Corthesy-Theulaz I, Dorta G, et al. Favourable effect of regular intake of fermented milk containing Lactobacillus johnsonii on Helicobacter pylori associated gastritis. Alimentary Pharmacology \& Therapeutics. 2003;18(8):805-813.

28. Chitapanarux T, Thongsawat S, Pisespongsa P, et al. Effect of Bifidobacterium longum on PPI-based triple therapy for eradication of Helicobacter pylori: A randomized, double-blind placebo-controlled study. Journal of Functional Foods. 2015;13:289-294.

29. Szajewska H, Horvath A, Kołodziej M. Systematic review with metaanalysis: Saccharomyces boulardii supplementation and eradication of Helicobacter pylori infection. Alimentary Pharmacology \& Therapeutics. 2015;41(12):1237-1245.

30. Wang F, Feng J, Chen P, et al. Probiotics in Helicobacter pylori eradication therapy: systematic review and network meta-analysis. Clin Res Hepatol Gastroenterol. 2017;41(4):466-475.

31. McFarland LV, Huang Y, Wang L, et al. Systematic review and metaanalysis: multi-strain probiotics as adjunct therapy for Helicobacter pylori eradication and prevention of adverse events. United European Gastroenterology Journal. 2016;4(4):546-561.

32. Chauviere G, Coconnier MH, Kernéis S, et al. Adhesion of human Lactobacillus acidophilus strain LB to human enterocyte-like Caco-2 cells. Microbiology. 1992;138(8):1689-1696.

33. Bruno G, Rocco G, Zaccari P, et al. Helicobacter pylori Infection and Gastric Dysbiosis: Can Probiotics Administration Be Useful to Treat This Condition? Can J Infect Dis Med Microbiol. 2018; 2018:6237239.

34. Du YQ, Su T, Fan JG, et al. Adjuvant probiotics improve the eradication effect of triple therapy for Helicobacter pylori infection. World $J$ Gastroenterol. 2012;18(43):6302. 